\title{
Sous-titrage et apprentissage des langues
}

\author{
Yves Gambier \\ Université de Turku
}

Our overview on subtitling and language learning is based on a fact and a question. The internationalisation of media and the development of Communication and Information technology (ICT) is bound to change our relationship to languages. But how can we improve our command of foreign languages? Memories and experiences of the classroom are not always positive. On the other hand, in today's world, we cannot avoid reading on screens. Since the beginning of the 1980's, several studies have been carried out on the possible use of intralingual subtitles for language acquisition. We first review the types of studies and the scope of some of them, before considering specific empirical studies dealing with different language competences (vocabulary, listening comprehension, language transfer). Finally, we suggest new research topics, regarding the use of subtitling in natural and institutional language learning settings.

\section{Mise en perspective}

L'internationalisation de la communication s'accélère grâce aux satellites et aux systèmes de réception qui les complètent, collectifs (réseaux câblés) ou individuels (antennes paraboliques). En outre, l'extension de la télévision numérique accroît le volume des programmes, sans oublier le rôle joué il y a peu par la vidéo et encore aujourd hui par les DVD, offrant des films traduits en plusieurs langues. Cette internationalisation des médias audiovisuels (AV), augmentés de la dissémination de l'Internet et des produits hors ligne (cédéroms, jeux vidéo), transforme les relations aux langues étrangères. Une certaine idéologie économique laisse accroire que l'anglais s'imposerait comme seule langue de cette évolution, que sa généralisation irait de pair avec le développement des technologies de l'information et de la communication (TIC), comme si l'internationalisation était synonyme d'uniformisation, comme si l'emploi d'une lingua franca pour quelques domaines et quelques segments de population rendait obsolète l'usage des langues vernaculaires, régionales ou locales (cf.Findhal 1989).

La logique de diffusion en réseau des médias permet de rassembler des audiences dispersées géographiquement et sociologiquement, reconfigurant les diasporas de naguère, les identités hybrides, mais aussi rendant plus visibles des communautés marginalisées, plus audibles des langues autrefois 'lointaines'. Les programmes de télévision, de radio - qu'ils soient distribués par satellite, câble ou sur le Net - sont devenus facteurs de proximité linguistique' tandis que certaines tâches et activités (achats, re- 
cherche d'informations et de documentation, jeux, etc.), désormais exercées à domicile, n'ont plus besoin d'intermédiaires. Les médias ainsi accessibles façonnent des besoins spécifiques - qu'on regarde une chaîne généralisée ou une chaîne thématique. Par ailleurs, l'environnement multimodal (visuel, graphique, chromatique, sonore, verbal, etc.) réintègre le langagier dans la complexité sémiotique: la langue n'est plus isolée des autres systèmes de signes, ce qui peut faciliter sa compréhension. Dans ce cas, le spectateur (apprenant ou pas) perçoit la langue (étrangère) dans un contexte culturel et intersémiotique.

En bref, l'internationalisation des médias, l'accroissement des volumes diffusés, l'accessibilité des langues, leur appréhension en contexte audiovisuel...construisent de nouvelles situations de communication, de nouveaux besoins langagiers et de nouvelles motivations d'apprentissage. Ce que certaines chaînes (BBC 1, 2 et BBC World en Grande-Bretagne, TV5 francophone, TV4 en Suède, etc.) ont très bien compris, en offrant des sous-titres intralinguistiques, pour aider les migrants, les apprenants...à renforcer leur maitrise en langue, quasiment en immersion. En plus, on ne lit pas seulement les écrans d'ordinateur au travail, mais également la télévision quand elle est sous-titrée, dans certains cas à plus de $80 \%$ des programmes aux heures de grande écoute (cas de la Finlande, par exemple).

Ce qui suit portera sur l'apprentissage des langues induit par le sous-titrage de programmes télévisés et de films, sur l'efficacité supposée de ce moyen. On ne traitera pas de l'enseignement ou cours de langues qui ont parfois leur place dans la grille des chaînes généralistes, ni des paroles défilantes de karaoké mises à la disposition d'enseignants de langues ou de lecture, ni des moyens AV développés pour promouvoir la lecture. L'aspect économique, lié à la distribution des produits AV sous-titrés et aux retombées éventuelles d'un apprentissage indirect, ne sera pas non plus abordé.

L'importance affirmée des sous-titres dans l'acquisition des langues étrangères exige encore des preuves empiriques. Néanmoins, elle a déjà donné lieu à des projets favorables au multilinguisme dans lesquels l'apprentissage des langues via le sous-titrage serait promu.

Ainsi l'Union Européenne (Commission 2005) s'est-elle engagée à étudier les possibilités d'utiliser davantage le sous-titrage interlinguistique pour développer le contact avec les langues et leur apprentissage, dans le cadre d'une politique en faveur de la diversité linguistique. Cet effort volontariste va à l'encontre des sondages Eurobaromètre sur les compétences linguistiques et les attitudes envers l'apprentissage des langues - sondages qui affirment que 60\% (décembre 2000) puis 56\% (décembre 2005) des Européens préfèrent regarder des programmes doublés. Ces pourcentages en fait confirment ce qu'on savait déjà, à savoir qu'on préfère ce à quoi on est habitué - d'où les réactions, confirmées par diverses enquêtes, des Allemands, Autrichiens, Espagnols, Italiens, Français penchant nettement pour le doublage au contraire des Nordiques, des Néerlandais, des Grecs, etc. 
Ainsi l'Afrique du Sud cherche-t-elle à combattre l'illettrisme et à développer ses onze langues officielles grâce à des programmes d'aide à la lecture et d'acquisition d'une seconde et troisième langues, dans lesquels le sous-titrage intralinguistique aurait un rôle à jouer (Kruger \& Rafapa 2002 et le présent volume). Il faut noter ici à la fois l'expérience menée en Inde de l'impact du sous-titrage sur l'alphabétisation de masse (Kothari 2000; Kothari et al. 2004) et la conviction de la Banque mondiale que le soustitrage peut être un des moyens les plus prometteurs pour éradiquer l'analphabétisme en Afrique. Ces projets, encore en latence ou déjà mis à l'épreuve, créent une demande pour vérifier les effets positifs du soustitrage sur la maitrise des langues (lecture en langue natale, compréhension en langues étrangères) - donnant de la sorte aux recherches déjà réalisées ou envisagées une pertinence sociale (sociolinguistique, socioculturelle, éducative).

\section{Types de problématiques et de travaux}

L'emploi de cassettes audio, de la vidéo, de films, de DVD dans les salles de cours, en particulier les classes de langue, a fait depuis longtemps l'objet d'interrogations, soulevant beaucoup d'espoir pour un renouveau pédagogique mais suscitant aussi beaucoup de questions, notamment pour les films. Faut-il visionner des extraits c'est-à-dire favoriser une approche sélective? Quels critères pourrait-on appliquer à la fois pour choisir un genre de long métrage et des séquences? A quelles activités (discussion, compréhension, prononciation, vocabulaire, registre de langue, initiation culturelle, etc.) peuvent servir ces séquences? Peut-il y avoir saturation à la projection et donc démotivation? Le sous-titrage peut-il aider à la création de tâches, d'exercices? Entrave-t-il l'écoute de la bande sonore ou rapproche-t-il lecture et audition? Appelle-t-il les mêmes réactions selon que les apprenants y sont exposés souvent ou pas?

Ces dernières questions sont à la base d'études essentiellement nord-américaines et européennes (donc avec des langues de même écriture romaine), souvent brèves, disparates dans leur teneur et leur objectif, portant surtout sur des groupes restreints d'apprenants de langue seconde (rares sont les exceptions comme Rogner 1992; Kothari 1999, 2000; Kothari et al. 2002, 2004). En outre, elles sont publiées dans des revues diverses portant sur différentes disciplines (éducation, pédagogie des langues, psychologie de l'enfant, psycholinguistique, linguistique appliquée, psychologie développementale, etc.). Un certain nombre de ces publications (depuis Price 1983) ont mis l'accent sur le sous-titrage intralinguistique (de même bande sonore que l'original), initialement utilisé par les sourds et connu aussi sous les noms de captions, same-language subtitling (SLS), "télétexte", "soustitrage bimodal" (passant du mode oral au mode écrit, pour la même langue) (Holobow et al. 1984; Lambert 1986; Danan 1992) ou "unilingue" (Vanderplank 1990). Pourtant dans ce qui suit, nous ne ferons pas référence 
à l'emploi de ce genre de sous-titre dans la formation linguistique des sourds.

Parmi les arguments pour recourir au sous-titrage intralinguistique, mis en avant dès le début des années 1980, on trouve:

- la forte présence de la redondance: nous percevons aussi le contenu, le sens à partir d'autres signes que linguistiques, ce qui est un atout pour ceux qui sont 'mal entendants' (hard of listening) c'està-dire qui ont des problèmes de concentration à l'écoute, de compréhension auditive (Holobow et al. 1984, sur des enfants anglophones de l'école élémentaire de niveau avancé en français);

- l'aide au décodage, à l'interprétation, ce qui est utile pour ceux qui ont un style d'apprentissage plus visuel qu'oral et pour tous ceux qui ne savent pas s'ils traitent l'information reçue à partir du son ou du texte (de Bot et al. 1986);

- l'amélioration de la compréhension orale (Price 1983; Vanderplank 1988; Markham 1989; Huang \& Eskey 1999).

Ces arguments ont engendré des contre-arguments, comme l'appréhension, notamment pour les apprenants les plus faibles, devant le défilement des sous-titres ressenti comme toujours rapide (cf. Vanderplank 1988) ou encore l'exigence de faire davantage d'effort et d'être davantage attentif pour vraiment bénéficier du médium AV (Salomom \& Leigh 1984). Ce n'est en effet pas parce qu'on est capable de lire des sous-titres qu'ils sont nécessairement ressentis comme agréables.

Les études proposées depuis plus d'une décennie sont souvent difficilement comparables au niveau des objectifs, des méthodes, et des résultats, et cela pour diverses raisons.

- Elles portent sur des langues et des directions de traduction différentes ${ }^{1}$, plusieurs solutions étant possibles :

Tableau 1

\begin{tabular}{|l|l|l|l|}
\hline & $\begin{array}{l}\text { Original } \\
\text { audio }\end{array}$ & $\begin{array}{l}\text { Sous- } \\
\text { titrage }\end{array}$ & L 2 : langue étrangère des apprenants \\
\hline 1 & L 1 & L 2 & $\begin{array}{l}\text { Sous-titrage interlinguistique dit inversé } \\
\text { (reversed subtitling) }\end{array}$ \\
\hline 2 & L 2 & L 1 & $\begin{array}{l}\text { Sous-titrage interlinguistique ordinaire, } \\
\text { conventionnel }\end{array}$ \\
\hline 3 & L 2 & L 2 & $\begin{array}{l}\text { Sous-titrage intralinguistique (bimodal L2 } \\
\text { input) }\end{array}$ \\
\hline 4 & L 1 & L 1 & $\begin{array}{l}\text { Sous-titrage intralingusitique (ex. TV5 } \\
\text { francophone) }\end{array}$ \\
\hline
\end{tabular}


1 et 3 servent pour un apprentissage délibéré (intentional learning) des langues tandis que 2 et 4 desservent plutôt une opportunité indirecte (incidental acquisition). ${ }^{2 \mathrm{i}}$

- Elles se déroulent plus souvent en milieu institutionnel, guidé (école, collège) qu'en milieu 'naturel' (quand on regarde la télévision chez soi) (cf. Pavesi \& Perego, à par). La fréquence d'exposition aux sous-titres, différente d'une culture à l'autre, selon les politiques traductionnelles des chaînes de télévision et des importateurs de films, influe évidemment sur leur réception (aisée ou inhibée) en classe.

- Elles mettent l'accent sur des compétences langagières variées de la part d'un apprenant qui n'est pas supposé être un récepteur passif (perception et reconnaissance phonétique, accents et variations sociolinguistiques, compréhension orale, vitesse de lecture, saisie et rétention de vocabulaire, et /ou traitement sémantique, stratégies de compréhension de l'information et d'inférence).

- Elles sont concernées par des niveaux d'acquisition ou périodes d'apprentissage différents, correspondant ou pas à des niveaux d'éducation précis (primaire, secondaire, universitaire, continue).

- Elles ont lieu sur des durées plus ou moins longues (de quelques jours à quelques mois), étant rarement longitudinales, comme si l'apprentissage d'une langue se faisait sur le court terme, et rarement comparatives (avec un groupe témoin ou parallèle ne recourant pas au matériel AV).

- Elles se développent à partir d'hypothèses et de concepts variés tantôt cognitifs (types de traitement de l'information, stratégies de lecture, types de mémoire: cf. Bird \& Williams 2002; Caimi 2006: 91-93), tantôt linguistiques (degré de maîtrise de tel ou tel élément, après soumission à un certain nombre de minutes ou d'heures de films sous-titrés), tantôt pédagogiques (théorie de l'apprentissage des langues; motivation et facteurs psychologiques d'apprentissage; styles et processus d'apprentissage).

- Elles s'interrogent à des degrés très divers sur le sous-titrage utilisé, produit par un professionnel en tenant compte des contraintes d'espace et de temps habituelles, ou produit à des fins didactiques ou expérimentales(Pavesi \& Perego, à par.). Le sous-titrage interlinguistique, plus condensé, plus périphrastique, moins littéral que celui intralinguistique, serait moins facilement employé dans les expériences et tests; il serait d'ailleurs la solution la moins bénéfique pour la compréhension en L2, comparé au sous-titrage inversé (la source en L1 aisément appréhendée, laisserait plus de temps pour traiter le texte en L2 qui exigerait de toute façon plus d'effort) et au sous-titrage bimodal (Lambert et al. 1981; Holobow et al. 1984; Lambert \& Holobow 1984; Koskinen et al. 1986; Lambert, 1986; Goldman \& Goldman 1988; Hawkins \& Myles 1986; 
d'Ydewalle \& Pavakanun 1989, 1996 a et b; Vanderplank 1988, 1990, 1993; Jung 1990; Pavakanun 1992; Danan 1992; Borrás \& Lafayette 1994). Dans presque tous les cas, on cherche à jouer sur la correspondance supposée quasi mot à mot, biunivoque, entre l'oral et l'écrit, la coexistence simultanée des deux codes (dual coding theory) améliorant, semble-t-il, la reconnaissance et la mémorisation lexicale, sinon la prononciation (pouvoir mnémotechnique du visuel) - voir section 3.1. A ce sujet, il est à remarquer que le sous-titrage intralinguistique destiné aux sourds et celui employé par quelques chaînes pour favoriser l'intégration linguistique de certaines couches de population ne suivent pas toujours les mêmes conventions (ainsi le premier doit indiquer tous les bruits et les effets prosodiques) et n'ont pas le même degré de littéralité - aucun cependant n'étant la copie conforme, exacte, complète (verbatim) de ce qui est dit. Répétons également que la plupart des études ont été réalisées dans des pays où le sous-titrage interlinguistique n'est pas forcément d'un usage familier.

\section{Etudes empiriques}

Dans ce qui suit, nous allons distinguer quelques travaux selon les compétences langagières visées. C'est un artifice de présentation, les méthodologies mises en place pouvant se croiser d'une recherche à l'autre d'une part, et les compétences n'étant pas étanches entre elles d'autre part (le vocabulaire aidant par exemple à la compréhension globale).

\subsection{Mémorisation et vocabulaire}

Entre l'analyse linguistique de versions doublées anglais-italien (Taylor 1996) ou anglais-allemand (Barbe 1996), incluant l'approche des registres de langue, des jeux de mots, des modalités, des interjections, des collocations, etc., et l'emploi des sous-titres surtout inversés ou bimodaux, l'éventail est assez large pour exposer des apprenants (en classe) à une langue étrangère. Bien des études ont démontré ainsi les effets positifs du sous-titrage intralinguistique sur la mémorisation et le rappel de mots, sur la réutilisation de vocabulaire contextualisé, sur les performances orales et écrites (Vanderplank 1988; Goldman \& Goldman 1988; Bean \& Wilson 1989; Garza 1991; Neuman \& Koskinen 1992; Danan 1992; Borrás \& Lafayette 1994; Koskinen et al. 1996; Baltova 1999; Markham 2004). A titre d'illustration, nous référons plus en détail à quelques-unes de ces expériences.

Garza (1991) a comparé l'aptitude à la compréhension de 70 apprenants d'anglais-langue seconde et de 40 étudiants américains en russe (3è et 4è années d'université). Après avoir visionné cinq extraits vidéo de 2-4 minutes, avec et sans sous-titres, les apprenants ont été soumis à des 
questions à choix multiples, portant sur des paraphrases, des déductions, des synonymes de termes en L2, visuellement explicités dans les extraits montrés. Les résultats démontrent une production améliorée, dans les deux groupes, quand il y a recours au sous-titrage. Le texte audio s'appuyant sur des images clarifierait donc le vocabulaire testé: les sous-titres rendraient les segments oralisés plus intelligibles, réduisant l'écart potentiel entre lecture et compréhension auditive.

Neuman et Koskinen (1992) confirment ces résultats positifs avec une batterie de tests plus grande (reconnaissance lexicale, identification de mots en contexte ou isolés, etc.), réalisés sur neuf semaines par 129 collégiens de niveau avancé en anglais (langue seconde), ayant regardé des extraits de 5 à 8 minutes d'un programme scientifique destiné aux jeunes Américains. Le sous-titrage intralinguistique était de nouveau bénéfique pour la reconnaissance et l'acquisition de vocabulaire.

Après avoir comparé trois méthodes d'approche (bande sonore en français (L2) seulement; sous-titres conventionnels en anglais L1; soustitres inversés en français, à partir de dialogues en anglais), Danan (1992: 506-518) a opté dans deux expériences menées auprès de 30 puis de 57 jeunes étudiants américains (respectivement de 2è et de 1è année en français) pour deux modes: le sous-titrage interlinguistique inversé et le sous-titrage intralinguistique bimodal (audio et écrit en français L2). Ses résultats, basés sur des tests à trou, confirment ceux de Lambert et al. (1981): après avoir regardé un extrait de cinq minutes d'une vidéo destinée à l'enseignement de la langue, les apprenants débutants démontrent une meilleure rétention lexicale avec les sous-titres inversés, la traduction ou plutôt la co-présence du visuel et des données bilingues, facilitant, semble-t-il, l'encodage (notamment la reconnaissance de la frontière des mots), l'assimilation et la mémorisation de la langue étrangère. Les résultats d'une troisième expérience (ibid.: 518-520), avec 15 apprenants plus avancés (toujours en français) vont dans le même sens. Globalement, le sous-titrage inversé apparaît comme la solution la plus bénéfique mais le sous-titrage bimodal a aussi des effets positifs, contrairement aux doutes exprimés par Lambert et Holobow (1984), selon lesquels les débutants ne pourraient pas s'appuyer exclusivement sur leur L2 dans leur apprentissage. Cette affirmation soulève donc la question récurrente dans l'enseignement des langues sur la place à accorder à la langue maternelle (L1) - voir section 3.3.

Bird et Williams (2002) ont également vérifié l'efficacité relative du sous-titrage bimodal sur l'acquisition de vocabulaire et pas seulement sur la compréhension, à partir de deux expériences incluant 16 natifs anglophones et des apprenants avancés en anglais (respectivement 16 et 24) et permettant d'évaluer leur temps de réaction (donc de décodage) devant des mots familiers ou non, leur mémoire implicite et leur mémoire (explicite) de reconnaissance.

Dans tous les cas cités ci-dessus, le choix des mots testés est justifié. En outre, il est fortement démontré que l'information phonologique dérivée à la fois d'une bande sonore et d'un écrit (sous-titres) contribue à 
améliorer le traitement des items parlés, renforçant l'idée que les systèmes cognitifs ayant affaire à la reconnaissance audio et visuelle des mots sont interactifs, interconnectés. Contrairement à une conception longtemps débattue, les processus phonologiques et orthographiques ne sont pas deux chemins distincts pour parvenir à la maîtrise lexicale. Entendre et lire simultanément aident les apprenants en langue étrangère.

\subsection{Compréhension orale}

La compréhension orale est un processus actif complexe qui s'appuie sur des connaissances antérieures, recourt aux inférences. Elle ne peut se réduire au seul décodage des phonèmes. Par contre, elle tire profit des signaux non linguistiques du contexte dans lequel elle prend place, notamment des éléments visuels. Depuis longtemps, les interprètes de conférence ont insisté sur l'importance de voir l'orateur pour mieux rendre son message, soulignant ainsi le rôle du non-linguistique dans la saisie de ce qui est dit et signifié. L'introduction des vidéoconférences, de l'interprétation à distance ont accrû le besoin de confirmer ces 'intuitions'.

Baltova (1994) a démontré avec 53 jeunes Canadiens en français langue seconde, de niveau intermédiaire, que les scores sont deux fois plus élevés pour la compréhension globale si on regarde un clip avec images et son plutôt que seulement le son. Cependant, les conclusions ne doivent pas être hâtives: jusqu'où le visuel aide-t-il à la compréhension d'un énoncé oral? Dans une deuxième expérience, l'auteure n'obtient pas de différence significative entre ses groupes. La vidéo (non sous-titrée) n'est pas une panacée.

Là encore, nombre d'études prouvent l'apport positif du soustitrage pour comprendre des détails touchant par exemple intrigue et personnages (Chiquito 1994-95; Chung 1999). Price (1983), Markham (1989, 1999), Nobili (1995), Vanderplank (1988, 1990 a et b, 1996a), Huang \& Eskey (1999) ont souligné, parmi bien d'autres chercheurs, le rôle du soustitrage intralinguistique pour améliorer la compréhension orale, dès lors qu'on a une assez bonne compétence en lecture.

Markham (1989) a testé, dans une université américaine, 76 étudiants en anglais langue seconde, mêlant les niveaux de compétence (débutant, intermédiaire, avancé). Tous, ayant regardé un court programme éducatif télévisé $\left(2 \frac{1}{2}-4 \frac{1}{2}\right.$ minutes), ont passé des tests de compréhension à choix multiple, portant à la fois sur des items lexicaux et syntaxiques présents dans les sous-titres intralinguistiques. Pour tous les niveaux, les réponses ont été plus correctes quand il y avait sous-titrage.

Dans une autre expérience, Markham (1999) va plus loin: il examine l'effet du sous-titrage intralinguistique sur la seule reconnaissance orale, indépendamment du composant écrit. Il soumet 118 étudiants avancés en anglais L2 à des tests de compréhension, réutilisant des phrases extraites de deux programmes vidéo de 12-13 minutes, suivies de quatre mots simples dont un seul est tiré de la phrase qui vient d'être énoncée. Selon les 
résultats, la présence des sous-titres accrôit l'aptitude des étudiants à identifier ces mots réellement prononcés et réentendus dans le test.

D'autres travaux confirment la corrélation entre sous-titrage et compréhension orale. Bird et Williams (2002), déjà cités, ont mis ainsi en évidence l'apprentissage implicite et explicite (mémorisation intentionnelle) des mots parlés et des pseudo-mots ou mots fabriqués (non-words). Le sous-titrage intralinguistique aide clairement la visualisation phonologique des éléments oralisés: les récepteurs sont moins troublés par les données ambiguës, gardent en mémoire une trace plus précise des mots et peuvent plus facilement, après un certain temps, identifier les sons identiques avec leur support textuel.

L'apport bénéfique de l'aide auditive que représente paradoxalement le sous-titrage a aussi été démontré par les quelques études à long terme menées à ce propos:

- $\quad$ par exemple Lambert et Holobow (1984) avec des élèves canadiens qui ont suivi un programme d'immersion en français depuis la maternelle: sur une période de 11 semaines, divers tests, mesurant sens contextuel et compréhension, prouvent que les sous-titres augmentent leur efficacité dans le temps dans le processus d'apprentissage de la langue;

- par exemple d'Ydewalle et van Rensbergen (1986) avec 58 enfants de niveau scolaire différent regardant deux dessins animés et s'interrogeant sur leur préférence, leur attitude, leur attention envers les sous-titres - les plus petits n'ayant pas fixé, stabilisé leur manière de lire les lignes en bas de l'écran;

- par exemple Vanderplank (1988, 1990b), avec des étudiants en anglais langue seconde (15 Européens et 8 Arabes) et de divers niveaux, regardant une heure de programme sous-titré par semaine (sur 9 semaines): très vite, ces apprenants ont admis pouvoir traiter de plus longues séquences verbales, remarquer les segments entièrement nouveaux pour eux (mots, propositions, collocations), extraire une certaine terminologie, reconnaître plus aisément accents, dialectes et humour;

- par exemple Van Rensbergen et al. (1986) ainsi que Koolstra et al. (1997).

De ce qui précède, on ne peut pas conclure que le sous-titrage intralinguistique est adapté pour toute sorte de matériel AV et pour tous les apprenants, indépendamment de leur niveau de compétence initiale (cf.Klinger 1993). Il est ainsi difficile d'imaginer que des sous-titres composés uniquement de tournures nouvelles soient utiles dans un apprentissage: l'exposition massive à des programmes sous-titrés, mal sélectionnés pour des non-natifs, peut être aussi un outil pédagogique inapproprié. Deux recherches sont à mentionner ici. D'abord celle de Lambert et Holobow (1984), portant sur de jeunes Canadiens ayant eu 45 minutes de français par jour, pendant sept ans 
à l'école: le sous-titrage intralinguistique bimodal est moins performant que le sous-titrage inversé (cf. section 2). La seconde recherche par Guillory (1998), testant 202 jeunes Américains débutants en français: les extraits vidéo, aux sous-titres surchargés et inadaptés ( $28 \%$ des mots utilisés n'étant pas listés dans le glossaire du manuel de la classe), ne pouvaient aider les apprenants. Dans ce cas, le sous-titrage ne compensait ni le débit rapide des énoncés ni le niveau de vocabulaire trop difficile. Il y aurait donc un seuil en deçà duquel le sous-titrage ne serait d'aucune aide ou d'une aide minimale, peut-être même contre-productive si elle bloque, inhibe l'apprenant. Un tel résultat converge avec les théories de l'apprentissage qui mettent en avant les facteurs affectifs, émotionnels et soulignent le rôle actif que doit jouer l'élève pour saisir, organiser, assimiler ce qu'il apprend.

\subsection{Avec le sous-titrage interlinguistique}

Les approches fonctionnelles et communicationnelles dans l'enseignement des langues tendent à exclure l'emploi de L1 dans les cours, même si certaines recherches sur les processus langagiers et la mémorisation montrent la validité d'un usage sélectif de L1 et des transferts entre L1 et L2 (Malmkjaer 1998). D'où les tentatives de recourir au sous-titrage interlinguistique, certes moins nombreuses que celles avec le sous-titrage intralinguistique. Pour certains, ce recours est vain sous prétexte que l'étudiant qui lit le texte en sa langue maternelle n'écoute plus la bande sonore en L2. Il est vrai qu'on n'échappe pas à la lecture des sous-titres (d'Ydewalle et al. 1985; d'Ydewalle et al. 1991; d'Ydewalle \& Gielen 1992: 416-417; d'Ydewalle \& Pavakanun 1991). Mais cette lecture quasi inévitable n'exclut pas le traitement de l'information orale: diverses expériences prouvent en effet qu'en l'absence de son, on s'attarde davantage au sous-titrage, comme si toute l'énergie cognitive devait se dépenser sur ce seul mode, faute de pouvoir se répartir sur l'audio et le visuel et d'alterner l'attention entre image/son et lecture (d'Ydewalle \& Pavakanun 1996; d'Ydewalle \& Gielen 1992).

De Bot et al. (1986) ont soumis deux groupes de jeunes (50 élèves du secondaire apprenant l'anglais et 20 étudiants avancés qui ne suivent plus de cours d'anglais L2) à un programme d'informations en anglais avec des sous-titres en néerlandais (L1) déviant parfois de ce qui était dit, soit au niveau grammatical, lexical, soit au niveau informationnel. Les résultats des tests à choix multiple, avec des questions également réparties entre ce qui divergeait et ce qui ne divergeait pas de l'original, indiquent clairement que les sources (orale, visuelle et écrite) sont simultanément traitées par les spectateurs, les chercheurs ne pouvant pas toutefois quantifier ce qui est appris des données audio.

L'apprentissage des langues via le sous-titrage interlinguistique est plus fréquemment indirect (incidental), sans qu'il semble y avoir un effort conscient ou systématique (d'Ydewalle \& Pavakanun 1989, 1996; Pavakanun \& d'Ydewalle 1989, 1992; van de Poel \& d'Ydewalle 1997; Koolstra \& Beentjes 1999 - toutes ces références portant sur la paire de langues 
néerlandais/anglais). D'où l'importance d'analyser plus en profondeur et méthodiquement cette acquisition éventuelle, reconnue très souvent dans les pays où le sous-titrage est la norme dominante à la télévision et au cinéma (cf. Vanderplank 1988: 276) - ce qui expliquerait par exemple l'aisance avec l'anglais chez des enfants (en Belgique néerlandophone, en Finlande, etc.) avant même qu'ils ne reçoivent un enseignement formel dans cette langue. Les adultes aussi, dans ces pays, admettent souvent que les sous-titres interlinguistiques justifient leur performance en L2 (de Bot et al. 1986, référant à un sondage de la télévision néerlandaise (NOS) réalisé en 1977). La familiarité de traiter des sous-titres pourrait également expliquer cette relative bonne maîtrise des L2: en classe, les élèves n'auraient aucune distraction ni anxiété devant l'offre bimodale et bilingue.

Blane (1996) a tenté d'intégrer un corpus de sous-titres interlinguistiques dans un programme de langue à l'université. En 2000, Williams et Thorne de Lampeter (Pays de Galles) ont, quant à eux, proposé de mettre sur pied un cours de sous-titrage comme moyen et occasion non pas de former des professionnels mais de développer les compétences langagières, en L1 aussi bien qu'en L2 - proposition validée par Neves (2004), Caimi (à par.) et Fernández (à par.) et par des projets comme LvS (Learning vis Subtitling) (Sokoli 2006) ou Streamline (Baldry 2002: 171-173). Rappelons que l'Union Européenne (Commission, 2005) se penche aussi sur les avantages éventuels d'un apprentissage 'naturel' grâce à ce type de sous-titrage (cf. section 1).

Parmi ces avantages, il faut mentionner encore les rares études centrées sur l'acquisition de la grammaire en L2, par le biais d'un programme télévisé sous-titré. Tandis que Van Lommel et al. (2006) se sont interrogés sur la réception de quelques règles syntaxiques (néanmoins après une présentation formelle) mesurée par des tests qui faisaient immédiatement suite à une courte projection AV, Ghia (à par.) s'est penché sur la maîtrise à long terme d'éléments comme l'ordre des mots, les traits morphosyntaxiques de certains verbes, les questions interro-négatives, l'ellipse, les dislocations à gauche et à droite, avec 22 jeunes adultes italiens. Après avoir visionné un film par semaine (sur 15 semaines), ces apprenants d'anglais (de niveau intermédiaire) ont subi une batterie de tests et rempli un questionnaire final, révélant leur effort conscient de prêter attention aux formes en L2. Le résultat global semble marquer un progrès en syntaxe, en dépit des variations selon les tâches et selon les individus. La question demeure de savoir si l'acquisition, dans ces deux travaux, est plus intentionnelle qu'accidentelle. Une autre question, soulevée par Ghia, a le mérite de mieux cerner l'emploi potentiel du sous-titrage interlinguistique: la mémorisation des sous-titres serait liée à leur 'simplification' syntaxique. D'où la nécessité de revenir au constat déjà formulé en section 2: les sous-titres considérés peuvent être fabriqués à des fins didactiques ou être repris tels que élaborés par des professionnels, avec leurs stratégies spécifiques, comme la simplification (cf. Pavesi 2002), la condensation, etc. (Gambier 2006). 


\section{Perspectives}

Quelles directions la recherche doit-elle maintenant prendre pour confirmer ou pas le rôle décisif des différents genres de sous-titrage dans la maîtrise d'une langue étrangère, pour valider ou pas certains résultats touchant la production ou la compréhension langagière? Klinger (1993) a critiqué qu'on puisse généraliser ce qu'on sait des bénéfices du sous-titrage à tous les étudiants en anglais langue seconde, plutôt qu'à ceux qui sont déjà avancés. Dans leur réponse, Neumann et Koskinen (1993), déjà prudents pourtant dans leur article de 1992, insistent sur l'essentiel, à savoir que même sans instruction formelle, on apprend une langue en regardant les programmes sous-titrés: l'apprentissage indirect ne peut être négligé ni sous-estimé. D'autres chercheurs, du même avis, vont plus loin pour renforcer cet atout: on peut préparer les étudiants à tirer encore un plus grand profit des sous-titres (intra- et interlinguistiques) en développant chez eux la conscience de certaines stratégies cognitives et métacognitives, comme par exemple savoir prédire le sens à partir d'éléments visuel (re)connus, apprendre à regarder un fragment de film, décider de se concentrer sur quelques données orales, accroître sa capacité d'auto-évaluation pour mieux identifier ses lacunes, savoir alterner son recours à l'oral, au visuel ou à l'écrit, etc. (Danan 2004: 74-75; Caimi 2006: 94-96). D'évidence, les apprenants non habitués aux sous-titres (standard) présenteront plus de difficultés au départ à voir leur avantage dans leur apprentissage de L2. Les études empiriques signalées, disparates et déjà datées, ont porté en général sur de petits échantillons de jeunes, observés sur d'assez courtes périodes. A un moment où les TIC, les multimédias sont en expansion, il serait temps de revenir sur certaines problématiques et de dépasser certaines limitations (cf. section 2), tout en reprenant les divers modes de combinaison L1-L2. Désormais les nouvelles générations d'apprenants sont pour la plupart des usagers du Net, des jeux vidéo, du téléphone mobile, etc., et n'ont pas les inhibitions de leurs prédécesseurs (testés dans les années 1980) sur le maniement des outils électroniques et sur la multimodalité des documents qu'ils reçoivent, parcourent, lisent.

On peut suggérer des travaux portant sur:

- les sous-titres inversés appliqués aux débutants;

- l'impact des sous-titres chez ceux qui regardent quotidiennement la télévision sous-titrée, notamment chez les petits qui peuvent déjà se sentir familiers avec l'anglais avant même d'aller à l'école;

- le long terme, selon qu'on apprend une langue en situation guidée ou 'naturelle';

- les différences entre les programmes sous-titrés à visée éducative ou pas;

- le rapport éventuel entre l'efficacité du sous-titrage et les styles d'apprentissage (visuel/verbal, inductif/ déductif, concret/abstrait, 
émotionnel/rationnel, synthétique/analytique, etc.) - styles qui peuvent révéler ou confirmer les différences entre sexes dans l'acquisition des langues;

- l'importance des sous-titres dans le développement de l'autodidactie ou au moins de l'autonomie d'apprentissage chez les adolescents et/ou chez les adultes, que ce soit pour la lecture ou les langues étrangères;

- les corrélations entre l'emploi de l'AV sous-titré et les facteurs affectifs, émotionnels de l'apprentissage, d'autant plus qu'on est plongé très tôt aujourd'hui dans un univers de multimédias.

Signalons pour finir que quelques thèses sont en cours (au Cameroun, au Portugal, en Finlande): elles analysent des langues et des situations différentes, marquant peut-être un renouveau de la problématique du sous-titrage dans l'acquisition des langues.

\section{Bibliographie}

Ayonghe, Suzanne (2006). Subtitling as a tool in Academic literacy programmes: the University of Buea. Projet de doctorat, University of Buea, Cameroon.

Baldry, Anthony (2002). "Multimodal concordancing and screen translation". Caimi (éd.) (2002), $161-175$.

Baltova, Iva (1994). "The impact of video on the comprehension skills of core French Students". The Canadian Modern Language Review 50, 507-532.

Baltova, Iva (1999). "Multisensory language teaching in a multidimensional curriculum: The use of authentic bimodal video in core French". Canadian Modern Language Review 56 (1), 3248.

Barbe, Katharine (1996). "Dubbing in the translation classroom". Perspectives. Studies in Translatology 4 (2), 255-274.

Bean, R.M \& R.M Wilson (1989). "Using closed captioned TV to teach reading to adults". Reading Research and Instruction 28 (4), 27-37.

Bird, Stephen \& John N. Williams (2002). "The effect of bimodal input on implicit and explicit memory: an investigation into the benefits of within-language subtitling". Applied Psycholinguistics 23, 509-533. http://www.cus.cam.ac.uk/ jnw12/subtitling.pdf

Blane, S. (1996). "Interlingual subtitling in the language degree". Sewell Penelope \& Ian Higgins (eds) (1996). Teaching translation in Universities: Present and future perspective. London:CITL,183-208.

http://www.univ.trieste.it/ didacticas/pagine/relazione.php?id=20041009084724.htm

Borrás, Isabel \& Robert Lafayette (1994). "Effects of multimedia courseware subtitling on the speaking performance on college students of French". The Modern Language Journal 78 (1), 61-75

Bravo, Conceição (à par.). Putting the reader in the picture: screen translation and foreignlanguage learning. Thèse de doctorat, Universitat Rovira i Virgili, Tarragona.

Caimi, Annamaria (éd.) (2002). "Cinema Paradiso delle lingue. I sottotitoli nell'apprendimento linguistico". Special issue of RILA-Rassegna Italiana di Linguistica Applicata 34, 1-2.

Caimi, Annamaria (2006). "Audiovisual translation and language learning: the promotion of intralingual subtitles". The Journal of Specialized Translation 6, July 2006, 85-98.

Caimi, Annamaria (à par.). "The educational benefits of intralingual and interlingual subtitling in the foreign language classroom”. J. Díaz-Cintas (éd.) (à par.).

Chiquito, A.B. (1994-1995). "Metacognitive learning techniques in the user interface: Advance organizers and captioning". Computers \& the Humanities 28 (4-5), 211-223. 
Chung, J. (1999). "The effects of using video texts supported with advance organizers and captioning on Chinese college students' listening comprehension: An empirical study". Foreign Language Annals 32 (3), 295-308.

Danan, Martine (1992). "Reversed subtitling and dual coding theory: New directions for foreign language instruction". Language Learning 42 (4), 497-527. Repris dans: B. Harley (éd.) (1995). Lexical Issues in Second Language Learning. Amsterdam \& Philadelphia: John Benjamins, 253-282.

Danan, Martine (2004). "Captioning and subtitling: Undervalued language learning strategies". Meta 49 (1), 67-77.

De Bot Kees, J. Jagt, H. Janssen, E. Kessels \& E. Schills (1986). "Foreign TV and language maintenance". Second LanguageResearch 2 (1), 72-82.

Diáz-Cintas, Jorge (éd.) (à par., 2008).The didactics of audiovisual translation.Amsterdam \& Philadelphia:John Benjamins.

d'Ydewalle Géry \& I. Gielen (1992). "Attention allocation with overlapping sound, image and text”. K. Rayner (éd.). Eye movements and visual cognition: Scene perception and reading. New York: Springer Verlag, 415-427.

d'Ydewalle Géry \& Ubolwanna Pavakanun (1989). "Acquisition of a second/foreign language by viewing a TV program”. Leuven, KUL, Laboratory of Experimental Psychology: Report 95. Repris dans: P. Winterhoff-Spurk (éd.) (1995). Psychology of Media in Europe.The state of the art, perspectives for the future. Opladen: Westdeutscher Verlag, 51-64.

d'Ydewalle Géry \& Ubolwanna Pavakanun (1991). "Watching foreign TV programs and language learning”. Leuven, KUL, Laboratory of Experimental Psychology: Report 128 Repris dans: F.Engel, D. Bouwhuis, T. Bösser \& Géry d'Ydewalle (éds) (1992). Cognitive modelling and interactive environments in language learning.Berlin: Springer Verlag, 193-198.

d'Ydewalle Géry \& Ubolwanna Pavakanun (1996a). "Le sous-titrage à la télévision facilite-t-il l'apprentissage des langues?". Y. Gambier (éd.) Les transferts linguistiques dans les médias audiovisuels. Villeneuve d'Ascq: PU du Septentrion, 217-223.

d'Ydewalle Géry \& Ubolwanna Pavakanun (1996a). "Could enjoying a movie lead to language acquisition?". Leuven, KUL, Laboratory of Experimental Psychology: Psychological Report 196. Repris dans: P. Winterhoff-Spurk, T.H. van der Voort (éds) (1997). New horizons in media psychology. Opladen: Westdeutscher Verlag, 145-155.

d'Ydewalle Géry, C. Praet, K.Verfaillie \& J. van Rensbergen (1991). "Watching subtitled TV. Automatic reading behavior". Communication Research 18 (5), 650-666.

d'Ydewalle Géry \& J. van Rensbergen (1986). "Developmental studies of text-picture interactions in the perception of animated cartoons with texts". Leuven, KUL, Laboratory of Experimental Psychology: Report 60. Repris dans: H. Mandl \& J.R. Levin (éds) (1989) Knowl edge acquisition from text and pictures. Amsterdam: Elsevier/North Holland, 233-248.

d'Ydewalle Géry, J. van Rensbergen \& J. Pollet (1985). "Reading a message when the same message is available auditorily in another language. The case of subtitling". Leuven, KUL, Laboratory of Experimental Psychology: Report 54. Repris dans: J.K. O’Reagan \& A. Lévy-Schoen (éds) (1987). Eye movements: From physiology to cognition. Amsterdam: Elsevier/North Holland, 313-321.

Fernández, Marco (à par.). "Using subtitled video materials for second language instruction”, in: J. Diáz-Cintas (ed.) (à par. 2008)

Findahl, Olle (1989). "Language in the age of satellite television". European Journal of Communication 4, 133-159.

Gambier, Yves (2006). "Le sous-titrage: une traduction sélective". J. Tommola \& Y.Gambier (éds) Translation and Interpreting. Training and Research. Turku : Turun Yliopisto, 21-37.

Garza, T. (1991). "Evaluating the use of captioned video materials in advanced foreign language learning". Foreign Language Annals 24 (3), 239-258.

Ghia, Elisa (à par.). "A case study on the role of interlingual subtitles on the acquisition of L2 syntax. Initial results". RILA - Rassegna Italiana di Linguistica Applicata (2007).

Goldman M. \& S. Goldman (1988). "Reading with closed-captioned TV". Journal of Reading 31 (5), 458-461.

Guillory, Gant H. (1998). "The effects of keyword captions to authentic French video on learner comprehension". CALICO Journal 15 (1-3), 89-108.

Hawkings, R. \& F. Myles (1986). "Does exposure to written French improve students performance in listening comprehension and oral reproduction? A pilot study". French Studies Bulletin 20, 1-3. 
Holobow Naomi. E., Wallace E .Lambert \& L.Sayegh (1984). "Pairing script and dialogue: combinations that show promise for second or foreign language learning". Language Learning 34 (4), 59-76.

Huang, Hsin-Chuang \& David Eskey (1999). "The effects of closed-captioned TV on the listening comprehension of intermediate English as a foreign language students". Journal of Educationa Technology Systems 28 (1), 75-96.

Jung, U. (1990). "The challenge of broadcast videotext to applied linguistics". IRAL 28 (3), 201219

Klinger, J.K. (1993). "Commentary: Comprehensible comments on Neumann and Koskinen”, Reading Research Quarterly 28 (4), 377-382.

Koolstra Cees M. \& Johanna W.J. Beentjes (1999). "Children's vocabulary acquisition in a foreign language through watching subtitled TV programs at home". Educational Technology Research and Development 47 (1), 51-60.

Koolstra, Cees M., T. van der Voort \& L. van der Kamp (1997). “TV's impact on children's reading comprehension and decoding skills. A three-year panel study". Reading Research Quaterly 31, 128-152.

Koskinen, Patricia, J. Knable, P. Markham, C. Jensema \& K. Kane (1996). "Captioned TV and the vocabulary acquisition of adult second language correctional facility residents", Journal of Educational Technology Systems 24 (4), 359-373.

Koskinen Patricia, R.M. Wilson \& C. Jensema (1986). "Closed-captioned TV: A new tool for reading instruction". Reading World 24 (4), 1-7.

Kothari, Brij (1999). "Same-language subtitling: Integrating post literacy development and popular culture on television". Media and Technology for Human Resource Development 11 (3), 111-117.

Kothari, Brij (2000). "Same-language subtitling on Indian television: Harnessing the power of popular culture for literacy". K.Wilkins (éd.) Redeveloping communication for social change: Theory, Practice and Power. New York: Rowman \& Littlefield, 135-146.

Kothari Brij, Avinash Pandey \& Anita R. Chudgar (2004). "Reading out of the 'idiot box': samelanguage subtitling on TV in India”. Information Technologies and International Development 2 (1), 23-44. www.mitressjournals.org/doi/pdf/10.1162/1544752043971170

Kothari, Brij, J. Takeda, A. Joshi \& A. Pandey (2002). "Same-language subtitling: A butterfly for literacy?". International Journal of Lifelong Education 21 (1), 55-66.

Kruger, Jan-Louis \& Lesibana Rafapa (2002). "Subtitling, literacy and education in South Africa: putting audio-visual media to work in the classroom". Etude présentée au congrès Languages and the Media. Berlin, 5-6 déc. 2002.

Lambert, Wallace E. (1986). "Dialogue in one language, sub-titles in another. Some alternatives for Learning languages". Joshua A. Fishman, Andrée Tabouret-Keller, Michael Clyne, B.

Krishnamurti \& M.Abdulaziz (éds) The Fergusonian Impact, vol. 1: From Phonology to Society. Berlin: de Gruyter, 479-516.

Lambert Wallace E., I. Boehler \& J. Sidoti (1981). "Choosing the language of subtitles and spoken dialogues for media presentations: Implications for second language education". Applied Psycholinguistics 2, 133-148.

Lambert Wallace E. \& N.E. Holobow (1984). "Combinations of printed script and spoken dialogue that show promise for beginning students of a foreign language". Canadian Journal of Behaviora Science/Journal canadien des sciences du comportement, 16, 1-11.

Malmkjaer, Kirsten (1998). Translation and Language Teaching. Manchester: St Jerome.

Markham, Paul (1989). "The effects of captioned TV videotapes on the listening comprehension of beginning, intermediate, and advanced ESL students". Educational Technology 29 (10), 38-41.

Markham, Paul (1999). "Captioned videotapes and second-language listening word recognition". Foreign Language Annals 32 (3), 321-328.

Markham, Paul (2004). "DVD captioned video and second language reading and listening comprehension". Etude présentée au congrès Languages and the Media. Berlin, 3-5 nov..2004.

Neuman Susan B. \& Patricia Koskinen (1992). "Captioned TV as 'comprehensible input': Effects of incidental word learning in context for language minority students". Reading Research Quarterly 27 (1), 95-106.

Neuman Susan \& Patricia Koskinen (1993). "On forests and trees. A response to Klinger". Reading Research Quarterly 28 (4), 383-385.

Neves, Josélia (2004). "Language awareness through training in subtitling”. Pilar Orero (éd.) Topics in Audiovisual Translation. Amsterdam \& Philadelphia: John Benjamins, 127-140. 
Neves, Josélia \& Rita Menezes Pinto (2006). "Translating television programmes for young audiences”. Etude présentée au congrès MUTRA, Multidimensional Translation. Copenhague, 1-5 mai 2006.

Nobili, Paola (1995). "Cinema(to)grafo. L'uso didattico dei sottotitoli per l'apprendimento delle lingue straniere". SILTA - Studi Italiani di linguistica teorica ed applicata, 24 (1), 151172.

Pavakanun, Ubolwanna (1992). Incidental acquisition of foreign language through subtitled television programs as a function of similarity with native language and as a function of presentation mode. Thèse de doctorat en psychologie, KULeuven.

Pavakanun Ubolwanna \& Géry d'Ydewalle (1989). "Acquisition of a second/foreign language by viewing a TV program”. Leuven, KUL, Laboratory of Experimental Psychology: Report 95. Repris dans: P.Winterhoff-Spurk (éd.) (1995). Psychology of Media in Europe.The state of the art, perspectives for the future. Opladen: Westdeutscher Verlag, 51-64.

Pavakanun Ubolwanna \& Géry d'Ydewalle (1992). "Watching subtitled television programs and language learning". International Journal of Psychology 27, 555 (abstract).

Pavesi, Maria (2002). "Sottotitoli: dalla semplificazione nella traduzione all' apprendimento linguistico". A. Caimi (éd.) (2002), 127-142.

Pavesi Maria \& Elisa Perego (à par.). "Interlingual subtitling as a means to enhance second languageacquisition”. J.Diaz-Cintas (éd.) (à par. 2008).

Price, Karen (1983). "Closed-captioned TV: An untapped resource", MATSOL Newsletter 12, 1-8.

Rogner, B.M. (1992). Adult literacy: Captioned videotapes and word recognition. Thèse de doctorat. The Cincinatti Union Institute.

Salomon, G \& T. Leigh (1984). "Predispositions about learning from print and television". Journal of Communications 34 (2), 119-135.

Sokoli, Stavroula (2006). "Learning via subtitling (LvS). A tool for the creation of foreign language learning activities based on film subtitling". Paper given at the Copenhagen conference Audiovisual scenarios, 1-4 May

Voir aussi: http://artemis.eap.gr/gr/eeyem/en_lvs.asp

Taylor, Christopher (1996). "The relevance of film dubbing and subtitling to language teaching". C.Heiss, R.M. Bolletieri Bosinelli (éds) Traduzione multimediale per il cinema, la televizione, la scena. Bologna: CLUEB, 271-277.

Van de Poel, Marijke \& Géry d'Ydewalle (1997). "Incidental foreign-language acquisition by children watching subtitled TV programs". Leuven, KUL: Report 221. Publié en 1999 dans Journal of Psycholinguistic Research 28, 227-244. Repris dans: Gambier Y., \& H. Gottlieb (éds) (2001). (Multi)Media Translation. Concepts, Practices and Research. Amsterdam \& Philadelphia: John Benjamins, 259-273.

Vanderplank, Robert (1988). "The value of teletext subtitles in language learning". English Language Teaching (ELT) Journal 42 (4), 272-281.

Vanderplank, Robert (1990a). "A (very) quiet revolution: English language learning through unilingual subtitled television”. Etude présentée au congrès international AILA. Amsterdam, août 1990

Vanderplank, Robert (1990b). "Paying attention to the words: Practical and theoretical problems in watching TV programmes with unilingual (Ceefax) subtitles”. System 18 (2), 221-234.

Vanderplank, Robert (1993). "A very verbal medium: Language learning through closed captions". TESOL Journal 3 (1), 10-14.

Vanderplank, Robert (1996a). "Effects of and effects with television in education: towards a strong hypothesis for the value of teletext subtitles/closed captions". Paper given at the CETaLL Symposium (Commission on Educational Technology and Language Lerning), AILA Congress, Jyväskylä, Finland, August 1996.

Vanderplank, Robert (1996b). "Really active viewing with Teletext subtitles and closed captions". Modern English Teacher 5 (2), 32-37.

Van Lommel S., A. Laenen \& Gery d'Ydewalle (2006). "Foreign-grammar acquistion while watching subtitled TV programmes". British Journal of Educational Psychology 76, 243-258.

Van Rensbergen J. \& Géry d'Ydewalle, N. Eeraerts \& G. Robben (1986). "A developmental study on reading subtitles: TV watching behaviour of children". Bulletin of the British Psychological Society 39, A 94.

Williams, Helen \& David Thorne (2000). "The value of Teletext subtitling as a medium for language learning”. System 28 (2), 217-228. 

${ }^{1}$ La directionalité en traduction et en interprétation est un sujet devenu récurrent depuis peu en
traductologie et dans la formation, alors qu'a dominé pendant longtemps le dogme que seul comptait le travail vers la langue maternelle (L 1).

${ }^{2}$ Un sujet abordé by Josélia Neves et Rita Menezes Pinto lors de leur présentation “Translating television programmes for young audiences", Congrès Mutra sur la Traduction

Multidimensionelle, Copenhague, 1- 5 mai 2006 